\title{
Invited Talk
}

\section{Wednesday 28th August 2019 \\ Transferring NLP models across languages and domains \\ Barbara Plank \\ IT University of Copenhagen}

\begin{abstract}
How can we build Natural Language Processing models for new domains and new languages?

In this talk I will survey some recent advances to address this ubiquitous challenge, from crosslingual transfer to learning models under distant supervision from disparate sources, multitasklearning and data selection.
\end{abstract}

\section{Short bio}

Barbara Plank is Associate Professor in Natural Language Processing at IT University of Copenhagen. She has previously held positions as assistant professor at the University of Groningen and the University of Copenhagen, and a postdoc position at the University of Trento. Her research interests within NLP are broad and include learning under sample selection bias (domain adaptation, transfer learning), learning from beyond the text and multimodal inputs, and in general learning under limited supervision for cross-domain and cross-lingual NLP, applied to a range of applications from author profiling, syntactic language understanding, information extraction and visual question answering.

Barbara is member of the advisory board of the EACL (European Association for Computational Linguistics) and publicity director of the Association for Computational Linguistics. 